\title{
A Pilot Study of Uterine Artery Embolization with Tris-Acryl Gelatin Microspheres in Guinea Pigs
}

\author{
Wen-Quan Zhuang • Guo-Sheng Tan • \\ Wen-Bo Guo $\cdot$ Jian-Yong Yang
}

Received: 2 April 2011/Accepted: 13 June 2011/Published online: 28 June 2011

(C) Springer Science+Business Media, LLC and the Cardiovascular and Interventional Radiological Society of Europe (CIRSE) 2011

\begin{abstract}
Objective This study was designed to establish guinea pigs as an animal model for uterine artery embolization (UAE) with tris-acryl gelatin microspheres (TAGM).

Methods Twenty-five female adult guinea pigs were randomly divided into two groups, including a uterine artery casting mould group $(n=10)$ and a UAE group $(n=15)$. Pelvic angiography and vascular casting mould were performed in the first group. The anatomical characters of the pelvic cavity in guinea pigs were described. In the second group, the technical feasibility of performing UAE with TAGM in guinea pigs was investigated. The histopathological slides of the uterus of guinea pigs after UAE were examined to inspect the outcomes of UAE.

Results The uterine artery springs from the internal iliac artery, ascends tortuously along the cervix, and gives off vertically $8-10$ branches to the cervix uteri and uterine horns. The diameters of the trunk of the uterine artery and its first branch were $0.32 \pm 0.027 \mathrm{~mm}$ and $0.14 \pm$ $0.01 \mathrm{~mm}$, respectively. For UAE animals, the dosages of $40-120$ and $100-300 \mu \mathrm{m}$ TAGM were $0.033 \pm 0.003 \mathrm{ml}$ and $0.015 \pm 0.002 \mathrm{ml}$, respectively. On histopathological slides, embosphere particles were found in the first branches of the uterine artery, the subserous arteries, and the intramural arteries. Inflammatory reactions in the uterus were common in guinea pigs after UAE. Local or dispersed
\end{abstract}

W.-Q. Zhuang · G.-S. Tan · W.-B. Guo $(\bowtie)$

Department of Interventional Radiology, The First Affiliated Hospital of Sun Yat-sen University, Guangzhou, Guangdong Province, China

e-mail: patrickguo2008@163.com

J.-Y. Yang

Department of Radiology, The First Affiliated Hospital of Sun

Yat-sen University, Guangzhou, Guangdong Province, China areas of necrosis in uterus also were observed in a few guinea pigs.

Conclusions Guinea pigs are an appropriate and feasible model for UAE with TAGM.

Keywords Guinea pig - Uterine artery embolization . Tris-acryl gelatin microspheres
Abbreviations
UAE Uterine artery embolization
TAGM Tris-acryl gelatin microspheres
DSA Digital subtraction angiography
IVC Inferior vena cava
HE Hematoxylin and eosin stain

\section{Introduction}

Uterine leiomyoma is the most common benign tumor of the female genital tract with an incidence rate of $20-40 \%$ among women of reproductive age [1]. Uterine artery embolization (UAE) is a minimally invasive technique, first reported by Ravina and colleagues in 1995 [2] and consistently proven to be an effective treatment of symptomatic uterine leiomyoma by many following studies [3-5]. Recently, it also has been suggested as an alternative treatment to hysterectomy and myomectomy [6-10].

However, it is still controversial whether UAE is safe for patients ho desire future fertility [3-5, 11-15]. The effect of UAE remains unclear due to the ethical difficulty in conducting prospective studies on human fertility. Previous studies of UAE on fertility mainly focused on ovarian function, pregnancy, abortion, and production. However, other factors also play important roles in fertility, 
such as the integrity of the endometrium. Therefore, more in-depth studies are required to reveal further the impacts of UAE on fertility.

Guinea pigs have been successfully developed as an animal model for uterine leiomyoma [16]. We attempted to investigate the impact of UAE on fertility using guinea pigs as an animal model. First of all, the possibility of technique of UAE in guinea pigs has to be answered. This was a pilot study to examine the use of UAE with tris-acryl gelatin microspheres (TAGM) in guinea pigs.

\section{Materials and Methods}

The study protocol was approved by the Laboratory Animal Ethics Committee at Sun Yat-sen University. This study was approved by Institutional Review Board at Sun Yat-sen University and was in accordance with the ethical standards of the responsible committee on human experimentation of Sun Yatsen University and with the Helsinki Declaration of 1975 . The study comprised two parts. In the first part, ten guinea pigs were used to study the anatomical characteristics of pelvic organs at necropsy, including the uterus, ovary, bladder, and vagina. Histological features of pelvic vessels, including the origin of the uterine artery and the origin and distribution of the internal iliac artery, also were examined by pelvic angiography. In addition, the lengths and diameters of the internal iliac artery, uterine artery, and its first branch were measured by the pelvic artery casting mould with a vernier caliper.

In the second part, bilateral UAE with TGAM was performed in 15 guinea pigs. The distribution of embolic agents in surgical specimens and histopathology of the myometrium and endometrium were observed.

\section{Animals}

Twenty-five female guinea pigs were housed individually with ad libitum access to food and water and a 12-h light/ dark cycle beginning at 6 a.m. The guinea pigs were 8 months old and weighed $600.8 \pm 40.5 \mathrm{~g}$.

\section{Anesthesia and Exposure of Pelvic Organs}

After overnight food deprivation, guinea pigs were sedated by intraperitoneal injection of chloral hydrate $(50 \mathrm{mg} / \mathrm{kg})$. Heart rate and oxygenation were monitored throughout the procedure using a pulse oximetry.

A longitudinal incision was made at the superior margin of the pubic symphysis. The intestinal canals were pushed away with pads to expose the pelvic organs, such as the uterus, bladder, and ovaries. The normal uterus, fallopian tubes, ovaries, and bladder were observed.
Uterine Angiography

The midinferior segment of the abdominal aorta was dissected and cannulated with a 26-gauge needle. The needle tube was fixed on the artery while the stylet was removed. Contrast media (UTRAVIST ${ }^{\circledR}$, Schering AG, Guangzhou, China) were injected into the artery through the stylet. Angiography of the abdominal aorta was performed with contrast media injection at a dose of $2.4 \mathrm{ml}$ and a velocity of $0.8 \mathrm{ml} / \mathrm{s}$, and digital subtraction angiography (DSA) was acquired at $3 / \mathrm{s}$. The pelvic arteries were observed using angiography.

\section{Uterine Artery Casting}

After the above-mentioned steps, guinea pigs were sacrificed with an overdose intravenous administration of sodium pentobarbital $(100 \mathrm{mg} / \mathrm{kg})$. The abdominal part of the inferior vena cava (IVC) was cut off. A consistent and slow perfusion through the remaining needle in the distal end of the cutoff point of IVC was performed until the effluent liquid from the proximal end of the cutoff point turned clear [15]. Then, perchloroethylene acetone solution dyed in red (200 g/l) was injected slowly into the abdominal aorta through the remaining needle with a 1-ml syringe. Additional perfusion at a dosage of $0.3 \mathrm{ml}$ was conducted every $1 \mathrm{~h}$ after the first perfusion and was repeated two to three times. All guinea pigs were kept at room temperature for $8 \mathrm{~h}$ to allow solidification of the perfusate. The origin and distribution of the uterine artery were observed under microscope at $\times 10$ magnification. When the perfusate was completely hardened, the guinea pigs were placed in $37 \%$ hydrochloric acid for $24 \mathrm{~h}$ to achieve corrosion. The specimens were washed in water and allowed to dry in air. The smaller branches disrupted during washing were repaired with the primary perfusate. The lengths and diameters of the iliac artery, uterine artery, and its branches were measured with a vernier caliper.

\section{UAE with TAGM}

\section{Preparation of Embolic Agents}

A total of $0.4 \mathrm{ml}$ of contrast media was combined with $0.2 \mathrm{ml}$ TAGM of $40-120 \mu \mathrm{m}$ diameter or $0.2 \mathrm{ml}$ TAGM of 100-300 $\mu \mathrm{m}$ diameter (Biosphere Medical, Rockland, MA), respectively, and shaken up for embolization.

\section{UAE}

Fifteen guinea pigs were anesthetized as described earlier. After the internal iliac artery was dissected, its origin was punctured with a 26-gauge needle. The needle tube was 
fixed with ligations, and the tip of the needle was inserted into the uterine artery. Angiography was performed to confirm the position of the tip. Contrast media were injected at a dose of $0.9 \mathrm{ml}$ and a velocity of $0.3 \mathrm{ml} / \mathrm{s}$, and the DSA acquisition rate was 3/s. A slipknot was tied in the bladder-perineum branch to temporarily block blood flow. Furthermore, in order not to embolize the vascular network of the ovary, the ovarian branch of the uterine artery was clamped temporarily with a microartery clamp. After the blood flow of the main trunk of the uterine artery slowed down, TAGM of 40-120- $\mu \mathrm{m}$ diameter were injected into the artery through the needle under $\mathrm{x}$-ray fluoroscopy. Subsequently, when the stasis or reflux of contrast media was observed in the main trunk of the uterine artery, 100-300 $\mu \mathrm{m}$ TAGM were injected to enhance embolization. After UAE, the slipknot in the bladder-perineum branch was untied to recover the blood flow, and the microartery clamp on the ovarian branch of the uterine artery was released. The same procedures were performed in the contralateral uterine artery. Finally, the incision site was sutured.

\section{Gross and Histopathological Examination of the Uterus}

All guinea pigs were killed 14 days after the UAE with an overdose intravenous administration of sodium pentobarbital $(100 \mathrm{mg} / \mathrm{kg})$. The uterus was removed in laparotomy and fixed in $10 \%$ formaldehyde. Histological samples were taken from four different transverse planes and four different longitudinal planes of the uterine horn and were prepared by routine hematoxylin and eosin (HE) staining. The uterus, including myometrium and endometrium, was examined grossly and histologically. The distribution of TAGM in arteries was observed under an optical microscope.

\section{Results}

The bilateral UAE procedure was successfully performed in all guinea pigs. Postoperative examination was daily conducted up to 14 days for signs of fever, loss of appetite, loss of weight, or lack of movement.

All guinea pigs survived the procedure without severe complications and resumed normal eating and drinking. No fever, infection, or bleeding was found in any guinea pig during the recovery period.

\section{Normal Uterine Anatomy and Pelvic Angiography in Guinea Pigs}

The uterus of guinea pigs was somewhat Y-shaped, with two separate uterine horns joined by a cylindrical inferior end (Fig. 1).

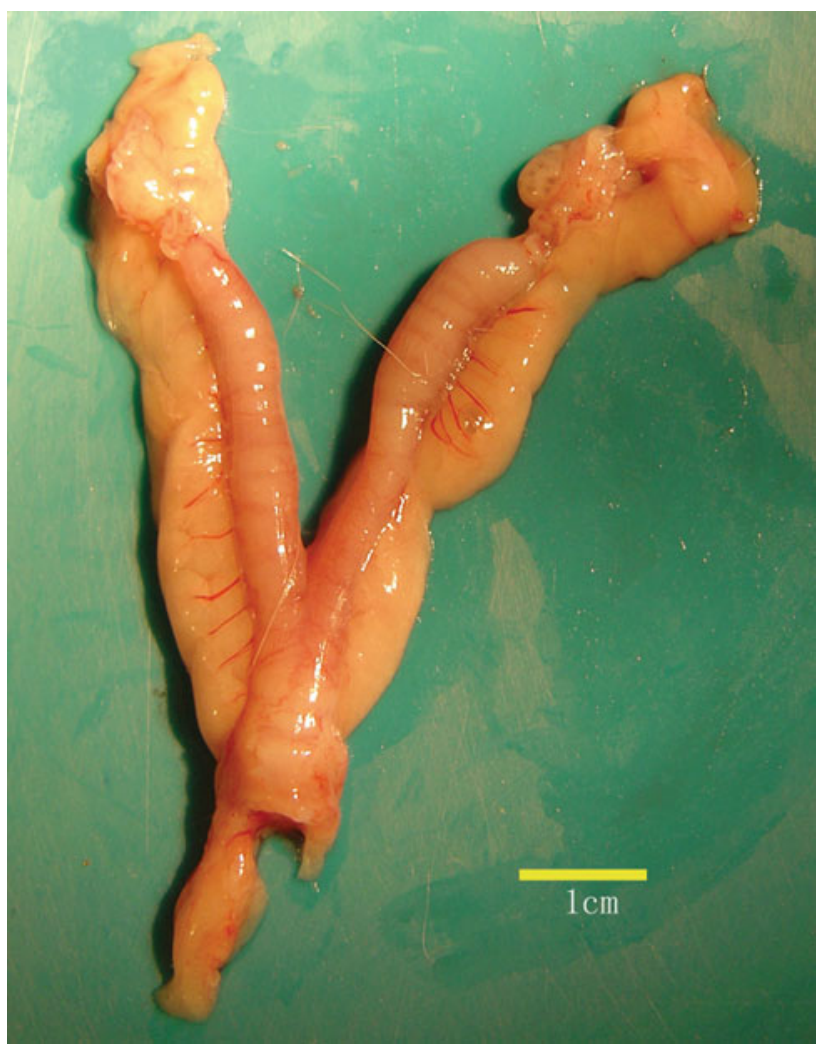

Fig. 1 Y-shaped uterus of guinea pigs

The angiography of the internal iliac artery demonstrated that the uterine artery springs from the ipsilateral internal iliac artery. The uterine artery ascends tortuously along the cervix and uterine horn and divides into $8-10$ vertical branches to the cervix uteri and uterine horns. It then turns laterally toward the hilus of the ovary and anastomoses with the ovarian artery. A bladder-perineum artery springs from the internal iliac artery and runs down to the bladder and perineum (Fig. 2).

\section{Vascular Casting}

The distribution of pelvic arteries in vascular casting was similar to that in angiography. The internal iliac artery was short and thick. The uterine artery rises windingly along the uterus and gives off branches into the cervix and uterine horns. The terminal portion twigs of the uterine artery divides into a small amount of vessels to the ipsilateral ovary (Fig. 3).

The Lengths and Diameters of the Uterine Artery and Its Branches

The lengths and diameters of the uterine artery and its firstlevel branches were measured in the vascular casting mould (Fig. 4). Results are summarized in Table 1. 


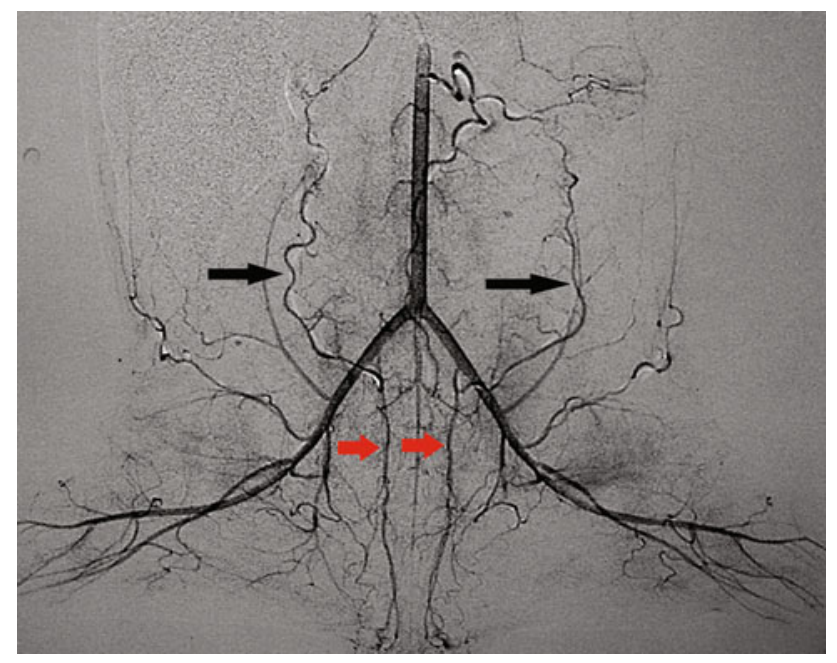

Fig. 2 Digital subtraction angiography (DSA) of pelvic arteries. Black arrow The uterine artery. Red arrow The bladder-perineum branch (Color figure online)

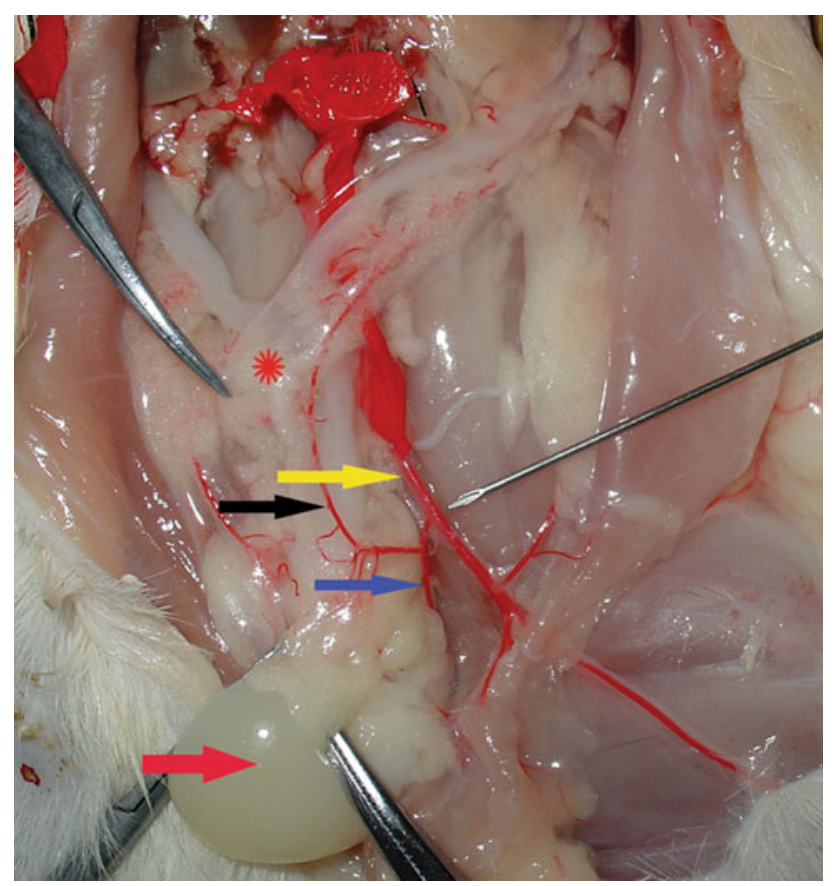

Fig. 3 Pelvic vascular casting mould. Black arrow The trunk of the uterine artery. Blue arrow The bladder-perineum branch. Yellow arrow Left common iliac artery. Red arrow Bladder. Red asterisk Uterus (Color figure online)

\section{UAE and Its Impacts}

The bilateral UAE procedure was successfully conducted in all guinea pigs. The uterine artery and its branches were distinctly visible in angiography before embolization and became invisible after embolization due to static contrast media (Fig. 5A, B). The dosage and usage of embolic agents are shown in Table 2. The embolization was

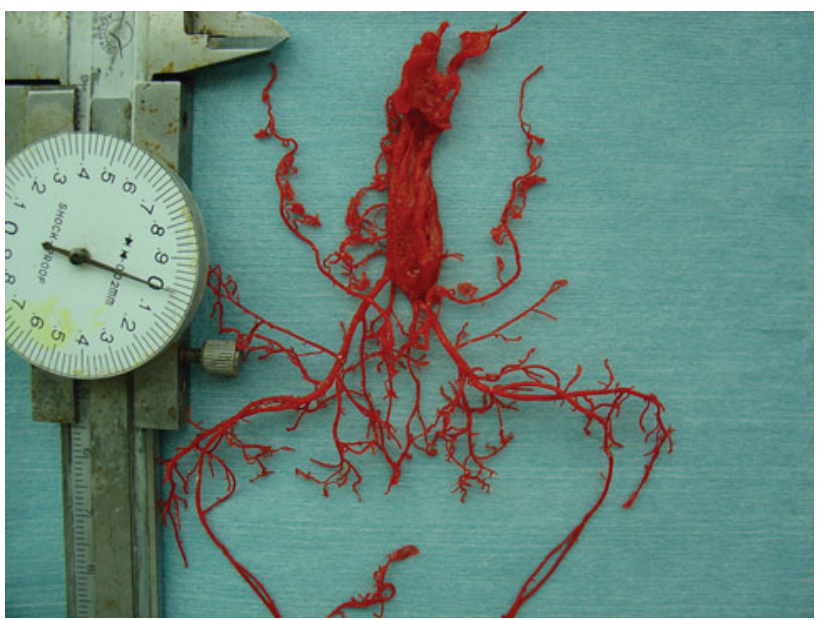

Fig. 4 Measurement of the uterine artery and its first-level branches in the vascular casting mould with vernier caliper

Table 1 Lengths and diameters of the internal iliac artery, uterine artery, and its first branch of guinea pigs (average \pm SEM, mm)

\begin{tabular}{lclc}
\hline & Iliac artery & Uterine artery & $\begin{array}{l}\text { First branch } \\
\text { of uterine artery }\end{array}$ \\
\hline Length $(\mathrm{mm})$ & $4.5 \pm 0.48$ & $42.4 \pm 2.36$ & $5.2 \pm 0.52$ \\
Diameter $(\mathrm{mm})$ & $0.48 \pm 0.042$ & $0.32 \pm 0.027$ & $0.14 \pm 0.017$ \\
\hline
\end{tabular}

satisfied without significant complications, because all guinea pigs were in good postoperative condition.

On day 14, all guinea pigs met the study protocol criteria for sacrifice. Animals were lapotomized, and the uterus, including both ovaries, was removed for gross and histological examination (summarized in Table 3).

At gross necropsy level, the ovaries, bowel, urinary bladder, liver, and spleen were normal. Nine of 15 guinea pigs seemed to have a normal uterus under gross examination. Further histological analysis showed that there was no degeneration or ischemic necrosis of the endometrium, and the construction of the myometrium and endometrium was clear. However, the uterus of all guinea pigs still suffered from inflammatory reactions. The remaining six guinea pigs had apparent gross and histological defects in the uterus. One guinea pig had local ischemic necrosis in the uterine horn with inflammatory reactions. Two guinea pigs suffered from edematous and congested uterus. For another two guinea pigs, their uterus showed a whitish coloration and ischemic changes under gross analysis and inflammatory-surrounded dispersed areas of necrosis under histological analysis. For the last guinea pig, a small amount of serosanguineous fluid and a few abscesses in the uterus were noted grossly. Under closer histological examination, its uterus had local necrotic tissue surrounded by inflammatory reactions. 
Fig. 5 A DSA before embolization. B DSA after embolization
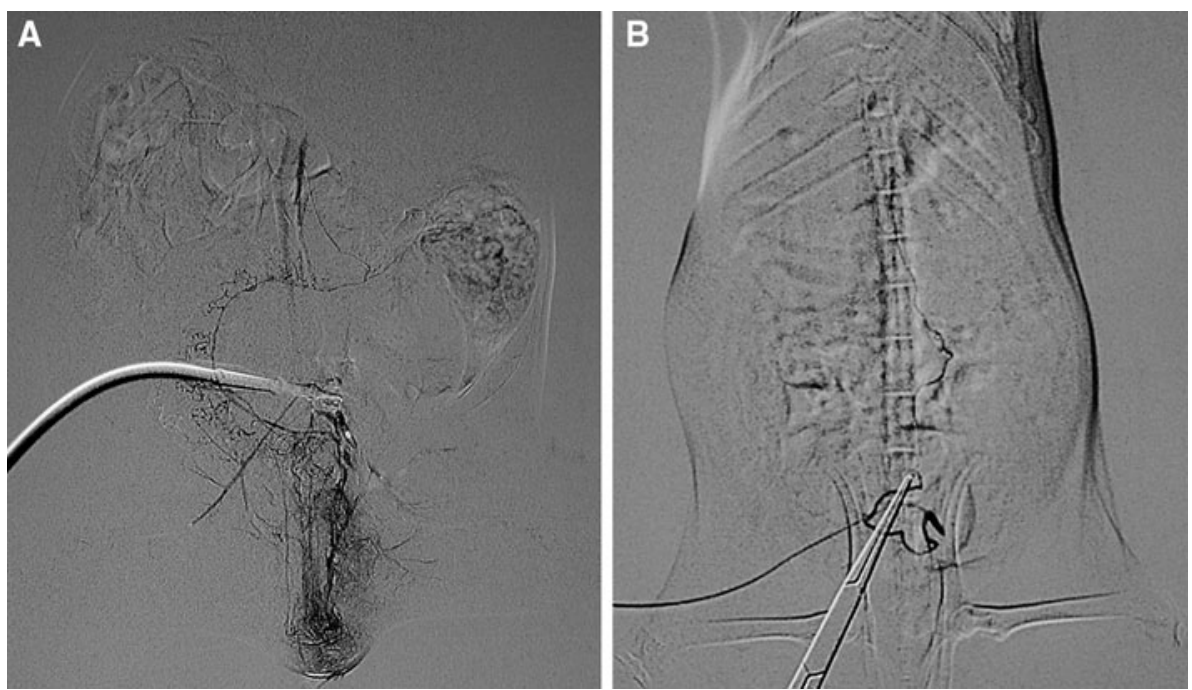

Table 2 Application and dosage of embosphere particles for embolization

\begin{tabular}{lll}
\hline $\begin{array}{l}\text { Diameter } \\
(\mu \mathrm{m})\end{array}$ & Application & $\begin{array}{l}\text { Dosage } \\
\text { (average } \pm \\
\text { SEM, ml) }\end{array}$ \\
\hline $40-120$ & $\begin{array}{l}\text { Embolization for the first branches and } \\
\text { smaller arteries of uterine arteries } \\
\text { Embolization for the trunks and the first } \\
\text { branches of uterine arteries }\end{array}$ & $0.031 \pm 0.003$ \\
$100-300$ & $0.015 \pm 0.002$ \\
\hline
\end{tabular}

Table 3 Gross and histopathological findings after the UAE procedure

\begin{tabular}{lll}
\hline Gross necropsy findings & Histopathologic findings & $\begin{array}{l}\text { No. of } \\
\text { guinea pigs } \\
(n=15)\end{array}$ \\
\hline $\begin{array}{l}\text { No obvious defects in the } \\
\text { uterus }\end{array}$ & $\begin{array}{c}\text { Edema, inflammatory } \\
\text { reaction, foreign body } \\
\text { Local ischemic necrosis } \\
\text { in the uterus }\end{array}$ & $\begin{array}{c}\text { Local necrosis in the } \\
\text { uterus, inflammatory } \\
\text { reaction, foreign body }\end{array}$ \\
$\begin{array}{c}\text { Edema and congestion in } \\
\text { the uterus }\end{array}$ & $\begin{array}{c}\text { Edema and hyperiaemia in } \\
\text { the uterus, inflammatory } \\
\text { reaction, foreign body }\end{array}$ & 2 \\
$\begin{array}{c}\text { Whitish coloration and } \\
\text { ischemic changes in } \\
\text { serous membranes } \\
\text { Local serosanguineous } \\
\text { fluid and abscesses in } \\
\text { the uterus }\end{array}$ & $\begin{array}{c}\text { inflammatory reaction, } \\
\text { foreign body } \\
\text { Local necrosis in the } \\
\text { uterus, inflammatory } \\
\text { reaction, foreign body }\end{array}$ & 2 \\
\hline
\end{tabular}

In addition, under histopathological examination, TAGM were observed in the uterus of all guinea pigs with routine HE staining. Embosphere particles were found in the uterine artery lumen of the first branches, the subserous arteries, and some of the intramural arteries (Fig. 6A-C). Embosphere particles, seen as red globules with welldefined boundaries, were distributed homogeneously. Erythrocytes and a small amount of lymphocytes were found around the embosphere particles.

\section{Discussion}

There are several advantages of choosing guinea pigs as an animal model for UAE with TAGM. Guinea pigs have been developed as an animal model for uterine leiomyomas with a high incidence rate of leiomyoma formation [16]. The biological behavior and histopathological features of leiomyomas in guinea pigs are similar to those in human beings [16]. In addition, its pregnancy duration (16 days) is shorter than sheep (150 days) and rabbit (32 days), making it easier to observe the impact of UAE on the uterus and ovary, and consequently on female fertility.

Because it is difficult to perform the UAE procedure by using a catheter in guinea pigs, we adopted the open surgical approach as previously described [17]. Our study investigated the technical feasibility to use guinea pigs as an animal model for UAE with TAGM in open abdominal surgery and measured the lengths and diameters of the uterine artery and its first branches for choosing the size of embolic agents.

There were two technical difficulties with the guinea pig model. First, the uterine arteries of guinea pigs are so thin and tortuous that it is difficult to cannulate arteries. Furthermore, the uterine artery runs along connective tissues close to the cervix uteri. Even when the uterine artery is exposed successfully under a surgical operating microscope, dissection is still time-consuming. Second, fixing the needle in the circuitous uterine artery is so challenging 
Fig. 6 A Embosphere particles in the lumen of the first branch of the uterine artery $(\mathrm{HE}, \times 40)$. B Embosphere particles in the subserosal artery (HE, $\times 40)$. C Embosphere particles in the intramural artery (HE, $\times 40)$
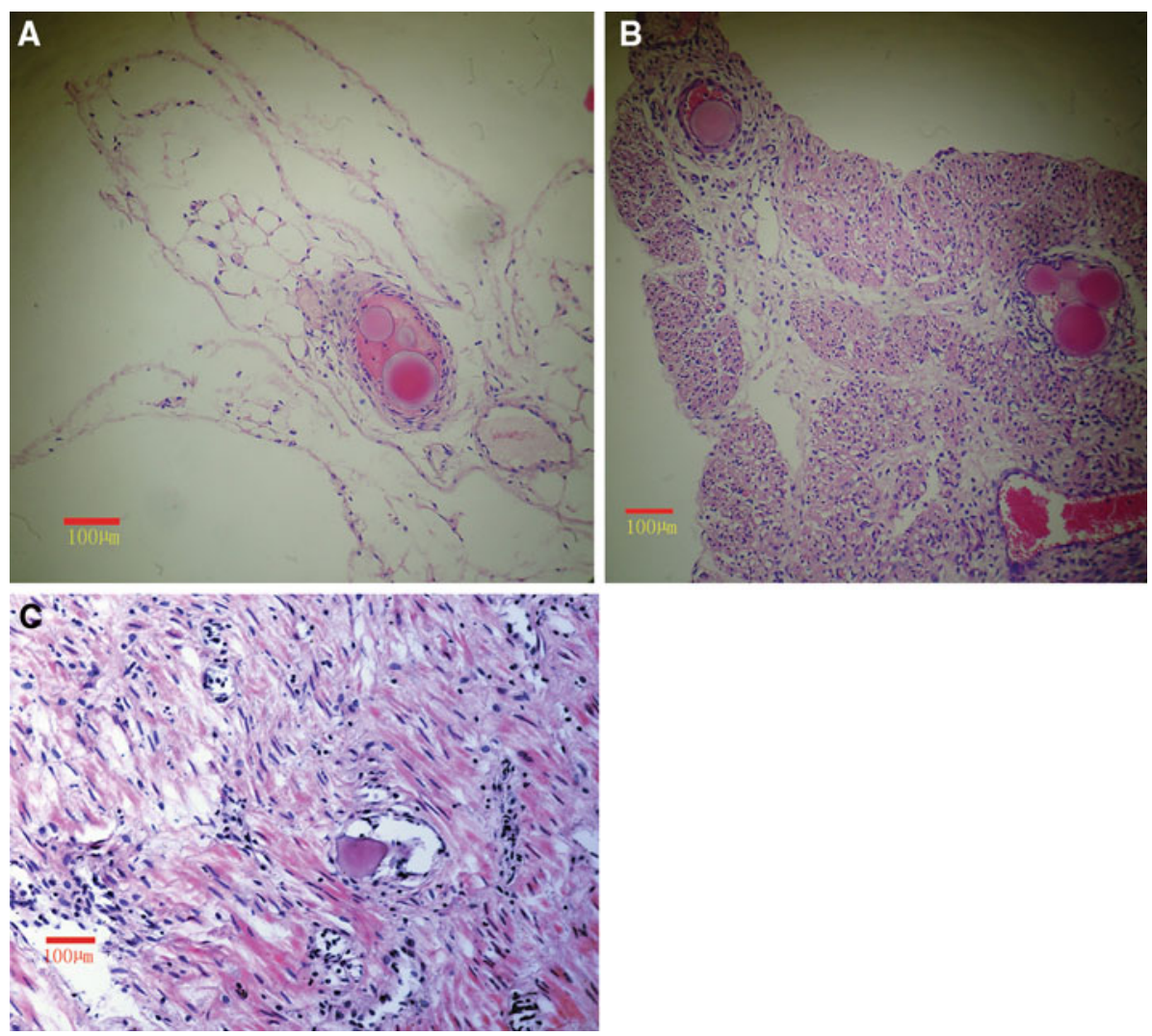

that it is almost impossible to control the embolization precisely. Moreover, due to the thin vessel of the uterine artery (only $0.3 \mathrm{~mm}$ in diameter), it is easy to cause vascular spasm and perforation during operation. Once these accidents happen, UAE can no longer be performed. Thus, the direct cannulation of the uterine artery has a low success rate.

In contrast with the uterine artery, the iliac artery is thicker (almost $0.5 \mathrm{~mm}$ in diameter) and diverges from the aortic abdominal artery at a large angle, making dissection and cannulation easier. Furthermore, only two major branches, namely the uterine artery branch and the bladderperineum branch, spring from the iliac artery in guinea pigs. When the bladder-perineum branch is blocked temporarily with a slipknot, the embolic agents only flow into the uterine artery, avoiding off-target embolization. In this study, we cannulated the iliac artery, maintained the tip of the needle close to the opening of the uterine artery, and blocked the bladder-perineum branch and the ovarian branch of the uterine artery temporarily. As a result, embolization of the ovary, bladder, and perineum was prevented. With this improved method, we successfully performed the UAE procedure in all guinea pigs. No ischemia or necrosis in the ovary, bladder, and perineum was found in any guinea pig. We believed that the UAE approach in our study is easy to operate and therefore has a higher success rate.
The diameters are approximately $0.3 \mathrm{~mm}$ for the main trunk of the uterine artery and $0.14 \mathrm{~mm}$ for the first branch in guinea pigs. To mimic the embolization procedure in humans as close as possible, we applied $40-120-\mu \mathrm{m}$ embolic agents to embolize the first branches and the artery network in uterus, and then injected a small amount of $100-300-\mu \mathrm{m}$ embolic agents into the main trunk of the uterine artery to enhance embolization.

In our study, the choice of embolic sizing was determined by two criteria. One was that the embolic particles had the potential to penetrate into the second branch or further vascular of uterine artery. This was for the next study of UAE for guinea pigs with uterine leiomyoma. On histopathological study after UAE, embolic agent particles spread in the cavities of the first branch of the uterine artery, the subserous arteries and some of the intramural arteries. It proved that the size of embolic agent met the criterion. Another was that the pathological change after UAE in guinea pigs was similar to that in humans as closely as possible. The pathological findings after UAE in humans demonstrated that the embolic particles were surrounded by a foreign-body giant cell reaction and acute inflammatory reaction. Furthermore, foci of myometrial necrosis away from the leiomyomas were found. In our study, inflammatory reactions were observed surrounding the embolic agents in the uterus without degeneration or necrosis in the myometrium and endometrium in most of 
the guinea pigs after UAE. There were local or dispersed areas of ischemic necrosis in the uterus of a few guinea pigs. Therefore, it met the second criterion, too. We believed that the size of embolic agent was appropriate for UAE in guinea pigs.

Under histopathological examination, embolic agent particles spread in the cavities of the first branch of the uterine artery, the subserous arteries, and some of the intramural arteries. In most guinea pigs after UAE, inflammatory reactions were observed surrounding the embolic agents in the uterus without degeneration or necrosis in the myometrium and endometrium. There were local or dispersed areas of ischemic necrosis in the uterus of a few guinea pigs. In humans, the embolic agents have been observed in the small blood arteries of myometrium [18]. Consistent with our study, the presence of inflammatory-surrounded embolic agents and areas of necrosis was noted in human uterine specimens [17]. Therefore, our pathological findings in guinea pigs are similar to those in humans. The sizes of embolic agents chosen in this study are proper for UAE in guinea pigs.

TAGM is a nonresorbable, deformable, and spherical embolic agents with calibrated sizes and uniform shapes. Due to its surface structure, deformability, and regularity of shape, it never clusters. So, it significantly penetrates deeper into the blood vessels than do PVA particles of either size. PVA particles tend to aggregate and thus occlude larger vessels. In terms of the characteristic of TAGM, it is easy to reach the goal of our study, which is to embolize the arterial network in uterus. The particles can penetrate the deeper vessels in uterus than do the other embolic agents through the uterine artery whose speed of blood flow was slow when we intubated iliac artery.

Sone et al. [19] mimicked the procedure of UAE in swine more closely to that of humans than we did. The choice of the GP sizes was determined by the size of embolic agents in humans. It would cause that the arterial distribution of embolic agents in swine after UAE was different from that in human, because the size of uterus in swine was different from that in human. In our study, the arteries of uterus in guinea pigs was measured first, and then it ensured that the arterial distribution of embolic agents in guinea pigs was similar to that in humans with the proper size of embolic agents according to the measurement of arteries of uterus in guinea pigs.

There are limitations of our study. First, the intubation by surgical exposure of iliac artery in guinea pigs is different from the clinical approach. Therefore, the arterial distribution of embolic agents in guinea pigs is not completely similar to that in humans because the embolic agents in guinea pigs could not run in uterine artery as it did in humans. So, it is inevitable that there were some bias in the results of our study. Second, a continuous observation of pathological changes of the uterus after UAE was not established in our study. The pathological change of uterus on the 14th day after UAE was observed only in our study. So, the description of acute inflammatory and necrosis in uterus was incomplete, but it was enough for the purpose of verifying the arterial distribution in uterus of embolic agents.

So far, there have been some researches with pig [20] and sheep [21] on UAE. However, these studies were flawed because that the animal models do not have uterine leiomyoma. So did our study. But this study is our pilot study on UAE for uterine leiomyoma in animal model. We will validate our technique of UAE on guinea pigs with uterine leiomyoma in our next study.

The embolization with TAGM ends when the contrast media are static in the uterine artery [22]. In the study by Belenky et al., methylene blue was injected intraprocedurally to determine the completion of UAE [23]. In our study, in contrast, angiography was used as an endpoint criterion and performed both before and after the UAE procedure, therefore more precisely controlling embolization. Our current study optimizes the operational procedures and paves ways for future studies on this animal model of uterine leiomyoma.

Acknowledgments The authors thank Dr. Xianghong Xiang for his assistance in casting pelvic arteries, and Dr. Ling Xue for her work on examining histopathological samples. Sources of financial support: initial plan of post-doctoral degree for medical research in Guangdong province [7300941] (the grant number is 30,000 RMB).

Conflict of interest All authors have not any potential conflicts of interest of a financial or other nature.

\section{References}

1. Sutton CJ (1996) Treatment of large uterine fibroids. Br J Obstet Gynecol 103:494-496

2. Ravina JH, Herbreeau D, Ciraru-Vigneron V et al (1995) Arterial embolization to treat uterine myomata. Lancet 346:671-672

3. Ravina JH, Amyrad A, Ciraru-Vigneron N et al (2003) Uterine fibroid embolization: results of 454 cases [in French]. Gynecol Obstet Fertil 31:597-605

4. McLucas B, Godwin S, Adler L et al (2001) Pregnancy following uterine fibroid embolization. Int J Gynecol Obstet 74:1-7

5. Goldberg J, Pereira L, Berghella V (2002) Pregnancy after uterine artery embolization. Obstet Gynecol 100:869-872

6. Pinto I, Chimeno P, Romo A et al (2003) Uterine fibroids: uterine artery embolization versus abdominal hysterectomy for treatment-a prospective, randomized, and controlled clinical trial. Radiology 226:425-431

7. Mara M, Maskova J, Fucikova $Z$ et al (2008) Midterm clinical and first reproductive results of a randomized controlled trial comparing uterine fibroid embolization and myomectomy. Cardiovasc Intervent Radiol 31:73-85

8. Goodwin SC, Bradley LD, Lipman JC et al (2006) UAE versus Myomectomy Study Group. Uterine artery embolization versus 
myomectomy: a multicenter comparative study. Fertil Steril 85:14-21

9. Siskin GP, Shlansky-Goldberg RD, Goodwin SC et al (2006) UAE versus Myomectomy Study Group. A prospective multicenter comparative study between myomectomy and uterine artery embolization with polyvinyl alcohol microspheres: long-term clinical outcomes in patients with symptomatic uterine fibroids. J Vasc Interv Radiol 17:1287-1295

10. Spies JB, Cornell C, Worthington-Kirsch R et al (2007) Longterm outcome from uterine fibroid embolization with tris-acryl gelatin microspheres: results of a multicenter study. J Vasc Interv Radiol 18:203-207

11. Abulafia O, Sherer DM (1999) Transcatheter uterine artery embolization for the management of symptomatic uterine leiomyoma. Obstet Gynecol Surv 12:745-753

12. Ravina JH, Vigneron NC, Aymard A et al (2000) Pregnancy after embolization of uterine myoma: report of 12 cases. Fertil Steril 6:1241-1243

13. Has R, Balci NC, Lbrahimglu L et al (2001) Uterine artery embolization in a 10 -week cervical pregnancy with coexisting fibroids. Int J Obstet Gynecol 72:253-258

14. Porter KB, Tsibris JCM, Nicosia SV et al (1995) Estrogeninduced guinea pig model for uterine leiomyomas: do the ovaries protect? Biol Reprod 52:824-832

15. Hardeman S, Decroisette E, Marin B et al (2010) Fertility after embolization of the uterine arteries to treat obstetrical hemorrhage: a review of 53 cases. Fertil Steril 94(7):2574-2579
16. Walker WJ, Barton-Smith P (2006) Long-term follow up of uterine artery embolisation-an effective alternative in the treatment of fibroids. BJOG 113:464-468

17. Colgan TJ, Pron G, Mocarski EJM et al (2003) Pathologic features of uteri and leiomyomas following uterine artery embolization for leiomyomas. Am J Surg Pathol 27:167-177

18. Fogt F, Hinds N, Zimmerman RL (2003) Histologic features of uterine leiomyomata treated with microsphere embolization. Obstet Gynecol 2003(102):600-602

19. Sone M, Osuga K, Shimazu K et al (2010) Porous gelatin particles for uterine artery embolization: an experimental study of intra-arterial distribution, uterine necrosis, and inflammation in a porcine model. Cardiovasc Intervent Radiol 33(5):1001-1008

20. Miyamoto S (2005) Uterine artery embolization using gelatin sponge in a miniature pig: a study of arterial size and the distribution of embolic materials. Nippon Igaku Hoshasen Gakkai Zasshi 65(4):452-454

21. Laurent A, Wassef M, Namur J et al (2010) Arterial distribution of calibrated tris-acryl gelatin and polyvinyl alcohol embolization microspheres in sheep uterus. Cardiovasc Intervent Radiol 33(5):995-1000

22. Worthington-Kirsch RL, Andrews RT, Siskin GP et al (2002) II. Uterine fibroid embolization: technical aspects. Tech Vasc Interv Radiol 5(1):17-34

23. Belenky A, Bartal G, Gat Y et al (2005) Uterine artery embolization: a pilot study in a rabbit model. Fertil Steril 83(2):487-490 